Full length article

\title{
How shape and internal structure affect the magnetic properties of anisometric magnetite nanoparticles
}

\author{
Helena Gavilán $^{\mathrm{a}, *}$, Oliver Posth ${ }^{\mathrm{b}}$, Lara K. Bogart ${ }^{\mathrm{c}}$, Uwe Steinhoff ${ }^{\mathrm{b}}$, Lucía Gutiérrez ${ }^{\mathrm{a}, \mathrm{d}, * *}$, M. Puerto Morales ${ }^{\mathrm{a}}$ \\ a Instituto de Ciencia de Materiales de Madrid, ICMM/CSIC, Cantoblanco, 28049 Madrid, Spain \\ ${ }^{\mathrm{b}}$ Physikalisch-Technische Bundesanstalt, 10587 Berlin, Germany \\ ${ }^{\mathrm{c}}$ UCL Healthcare Biomagnetics Laboratory, University College London, 21 Albemarle Street, London W1S 4BS, UK \\ d Dept. Química Analítica, Instituto de Nanociencia de Aragón, Universidad de Zaragoza, 50018 Zaragoza, Spain
}

\section{A R T I C L E I N F O}

\section{Article history:}

Received 27 September 2016

Received in revised form 9 December 2016

Accepted 11 December 2016

Available online $\mathrm{xxx}$

\section{Keywords:}

Nanoparticles

Magnetic properties

Size effects

Shape dependence

Structure properties relationship
A B S T R A C T

A three-step aqueous approach to obtain large $(>50 \mathrm{~nm})$ magnetite single-core particles has been developed. The steps are a) synthesis of antiferromagnetic nanoparticles, b) particle coating and c) subsequent reduction of the core material to magnetite. By variation of precursor material and process conditions, the synthesis yielded rhombohedra, discs or needles below $200 \mathrm{~nm}$. A combination of X-ray diffraction, ${ }^{57} \mathrm{Fe}$ Mössbauer spectroscopy and infrared spectroscopy confirmed magnetite to be the dominant final core material. From transmission electron microscopy, we identified porous structures after the reduction. Magnetic characterization of the different magnetic nanopaticles revealed strikingly different magnetic behaviour depending on their shape, internal structure and reduction process. We conclude that each of these parameters have to be considered in further characterization of large magnetite nanoparticles.

(C) 2016 Published by Elsevier Ltd.

\section{Introduction}

In the last years, iron oxide magnetic nanoparticles (MNPs) have been widely used for biomedical applications. Examples of these applications include new ways of cancer treatment such as magnetic drug targeting [1] and magnetic hyperthermia [2], or the use of MNPs as contrast agents or tracers in Magnetic Resonance Imaging [3] and Magnetic Particle Imaging [4]. Each application requires MNPs with customized structural and magnetic properties, which are strongly dependent upon both particle size and shape [5]. As an example, ultra-small superparamagnetic MNPs with magnetic core sizes below $10 \mathrm{~nm}$ were suitable for the exploration of tumour permeability [6]. However, the uptake of MNPs by macrophages was most effective for MNPs with a core size of 10-30 nm, while for magnetic hyperthermia, maximum heating rate is obtained at the transition from multidomain to single domain magnetic behaviour occurring in a relatively broad size range at about $30 \mathrm{~nm}$ with a change of transition of magnetic energy into thermal energy (Néel or Brown) [7]. Moreover, if collective magnetic behaviour of the cores exists originating from interactions within a nanoparticle, heating rates can go up to one order of magnitude higher than for single-domain nanoparticles [8,9].

\footnotetext{
* Corresponding author.

** Corresponding author. Instituto de Ciencia de Materiales de Madrid, ICMM/CSIC, Sor Juana Inés de la Cruz 3,Cantoblanco, 28049 Madrid, Spain.

Email address: helena_gr@icmm.csic.es (H. Gavilán)
}

Nanoparticle shape can also have significant impact on the magnetic behaviour of MNPs in applications. Firstly, the magnetic shape anisotropy of the MNPs can assume much larger values than the magnetocrystalline anisotropy and thus can strongly affect the orientation of magnetic moments inside the particles. In addition, dipolar interactions between MNPs also depend on the particle shape and will influence the structural agglomerate formation leading to, for example, chain-like arrangements biomimicking magnetotactic bacteria [10] or hollow spheres consisting of oriented aggregates of nanocrystals $[11,12]$.

One of the current challenges in nanoparticle research is the production of particles [13] comprising a large magnetite core that is well-controlled in size and shape, with a large magnetic moment and long term colloidal stability. Over the past decades, direct syntheses of magnetite NPs with different sizes and morphologies have been reported, including nano- spheres [13], cubes [14,15], wires [16], rods [17], octahedral [18], plates [19] and prisms [20]. Synthesis of each of these shapes was performed out in organic media, so that, in general, nanocrystal shape control was achieved by selective adhesion of surfactant to a particular crystal facet and this subsequent slow growth along this direction. Without surfactants, nanoparticles can aggregate into dense or hollow micrometre spheres $[11,12]$. Synthesis in organic media is limited by the small particle batches that require further steps to be transferred to water, which results in low yields.

In the present paper, we describe an alternative aqueous-based approach to produce single-core magnetite MNPs with different morphologies and core sizes above $25 \mathrm{~nm}$, which could be scalable for 
large production. Magnetic iron oxide nanorods were already produced by a similar method and showed interesting magnetic properties and tuneable surface functionality [21]. We propose a three-step process (Fig. 1) from which uniform rhombohedra, discs and elongated MNPs can easily be obtained. First, an aqueous synthesis route is followed to obtain uniform antiferromagnetic precursors such as goethite or hematite, whose size and shape can be tuned by changing the synthesis conditions including temperature, pressure, and nature and concentration of the salts used [22]. Then, the antiferromagnetic precursor particles are coated by a silica layer that prevents their aggregation [23,24]. Finally, the silica coated antiferromagnetic particles are reduced to magnetite. This is either performed on particles in powder form (dry-reduction), by exposing them to a hydrogen atmosphere at a certain partial pressure [25], or in liquid form, using oleic acid and an organic solvent (wet-reduction). In both processes, the hydrogen and oleic acid act as the reducing agents [26,27]. Transformation of these antiferromagnetic phases into magnetite particles requires the full control of key parameters including temperature, atmosphere and pressure to produce pure single phases and avoid core sintering.

The products are physicochemically characterized by electron microscopy, X-ray diffraction, ${ }^{57} \mathrm{Fe}$ Mössbauer spectroscopy and infrared spectroscopy. Their magnetic behaviour is analysed by field and temperature dependent magnetization measurements. This characterization allows for comparing the products of dry reduction and wet reduction.

\section{Experimental}

\subsection{Synthesis of the precursors}

The preparation of uniform and nanometer precursor particles requires slight modifications of reported synthetic routes for hematite [26] and goethite [28] to obtain particle sizes below $200 \mathrm{~nm}$ and keep the uniformity.

(i) Hematite: The synthesis of hematite $\left(\alpha-\mathrm{Fe}_{2} \mathrm{O}_{3}\right)$ nano-sized rhombohedra and discs was carried out in a glass bottle dissolving $\mathrm{FeCl}_{3} \cdot 6 \mathrm{H}_{2} \mathrm{O}$ in $50 \mathrm{~mL}$ of pure ethanol and $10 \mathrm{~mL}$ or $1 \mathrm{~mL}$ of water for rhombohedra or discs respectively under vigorous magnetic stirring. Then sodium acetate was added while magnetically stirring and the solution was homogenized by sonication for $10 \mathrm{~min}$. The final solution had a concentration of $\mathrm{FeCl}_{3}$ of 0.082 and $0.096 \mathrm{M}$ for rhombohedra and discs, respectively. The concentration of sodium acetate was 0.49 and $0.58 \mathrm{M}$ for rhombohedra and discs, respectively. The mixtures were sealed in a teflon-lined autoclave $(125 \mathrm{~mL})$ and maintained at $180^{\circ} \mathrm{C}$ for $12 \mathrm{~h}$ for solvothermal crystallization. After natural cooling to ambient temperature, the resulting red solid product was washed with dis tilled water, filtered and finally dried overnight in an oven at $50{ }^{\circ} \mathrm{C}$.

(ii) Goethite: The synthesis of goethite $(\alpha-\mathrm{FeOOH})$ nano-sized needles was performed by precipitation of a $\mathrm{Fe}(\mathrm{II})$ sulfate $0.15 \mathrm{M}$ aqueous solution by the addition of a $0.225 \mathrm{M}$ solution of sodium carbonate. The resulting dispersion was then oxidized at constant temperature. The concentration ratio of the reagents $\left[\mathrm{CO}_{3}{ }^{-}\right] /$ $[\mathrm{Fe}(\mathrm{II})]$ is 1.5 . These processes were carried out according to the following procedure. Oxygen was first removed from the distilled water by $\mathrm{N}_{2}$ bubbling to prevent $\mathrm{Fe}(\mathrm{II})$ oxidation during the dissolution of $\mathrm{FeSO}_{4}$. The $\mathrm{Fe}(\mathrm{II})$ and sodium carbonate solutions $125 \mathrm{~mL}$, each) were prepared with the desired concentrations. The sodium carbonate solution was then introduced into a thermostatic water bath at $44{ }^{\circ} \mathrm{C}$ under moderate stirring and the $\mathrm{Fe}(\mathrm{II})$ solution was added applying a constant air flow with a rate of 2 $\mathrm{Lmin}^{-1}$ through the resulting suspension. Particles of goethite are then formed by aerial oxidation. After $180 \mathrm{~min}$ of reaction, the resulting precipitate was cooled, centrifuged and washed several times with distilled water. Finally, the powdered solid was collected by filtration and dried overnight at $50{ }^{\circ} \mathrm{C}$.

\subsection{Coating and reduction to magnetite}

Silica coating and subsequent reduction of the precursor particles were carried out by optimizing procedures described elsewhere [25-27]. Silica coating ensures both, steric and electrostatic protection and it acts as dispersing agent of many electrostatic colloids. These advantages render silica an ideal, low-cost material to tailor surface properties. Additionally, this coating should endow the cores with several beneficial properties, such as the biocompatibility and the possibility of subsequent functionalization by connecting the silanol groups on the surface with other organic molecular agents [29].

For the silica coating, $100 \mathrm{mg}$ of the precursor particles in powder form were dispersed in a 2-propanol/ $\mathrm{H}_{2} \mathrm{O} 2: 1 \mathrm{v} / \mathrm{v}$ solution of $300 \mathrm{~mL}$ $\mathrm{NH}_{4} \mathrm{OH} 28 \% \mathrm{v} / \mathrm{v}$ and tetraethyl orthosilicate (TEOS) were added during sonication and the reaction was allowed to continue for $15 \mathrm{~min}$. The final volume was $320.2 \mathrm{~mL}$ and the final concentration of the reagents was: $\left[\mathrm{NH}_{4} \mathrm{OH}\right]=4.5 \times 10^{-4} \mathrm{M},[\mathrm{TEOS}]=2.8 \times 10^{-6} \mathrm{M}$. The product was washed twice with 2-propanol. The solution was filtered and dried overnight at $50{ }^{\circ} \mathrm{C}$.

(i) Wet reduction: For the reduction to magnetite in liquid, $50 \mathrm{mg}$ of the silica coated precursor nanoparticles and $35 \mathrm{~mL}$ of trioctylamine were mixed by sonication for $30 \mathrm{~min}$ and subsequently $2.2 \mathrm{~mL}$ oleic acid were added. The mixture was transferred into a three-necked flask and heated at $350{ }^{\circ} \mathrm{C}$ under $\mathrm{H}_{2}$ flow of 0.486 $\mathrm{Lmin}^{-1}$ for $1 \mathrm{~h}$ until the colour changed to dark. After cooling to ambient temperature, the black product was collected by centrifugation and washed 3 times with toluene. Finally, the product was dried overnight at $50{ }^{\circ} \mathrm{C}$.

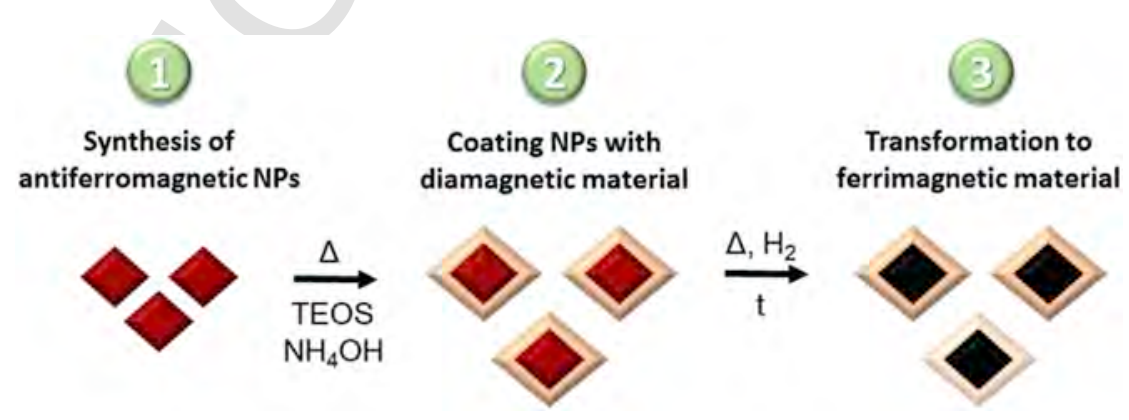

Fig. 1. General scheme for the synthesis of single-core MNPs. 
(ii) Dry reduction: For dry reduction, $50 \mathrm{mg}$ of the silica coated precursor nanoparticles in powder form were treated at $360{ }^{\circ} \mathrm{C}$ for $2 \mathrm{~h}$ in vacuum to remove moisture. The reduction was performed under $\mathrm{H}_{2}$ atmosphere with a partial gas pressure of $61 \mathrm{kPa}$ for $3.5 \mathrm{~h}$ refreshing the $\mathrm{H}_{2}$ atmosphere after $1 \mathrm{~h}$. The sample was then cooled down to ambient temperature.

\subsection{Nanoparticle characterization}

The size of the iron oxide cores in both the precursors and the final products was determined by transmission electron microscopy (TEM) with a JEM1010 microscope (JEOL, Peabody, USA) operating at $100 \mathrm{kV}$. TEM samples were prepared by placing a drop of the particles suspended in water onto a carbon coated copper grid and allowing it to dry at room temperature. The size distribution was determined by manual measurement of more than 100 particles using the public domain ImageJ software (http://imagej.nih.gov/). High-resolution TEM (HRTEM) was performed in a JEM-3100 F microscope (JEOL, Peabody, USA) operating at $300 \mathrm{kV}$. The particle morphology was determined from scanning electron microscopy (SEM) with a Philips XL30 microscope operating at $10 \mathrm{kV}$. The sample preparation is identical to the preparation for the TEM analysis.

The crystal structure of the antiferromagnetic precursors and the reduced particles was identified using a combination of X-ray diffraction (XRD), Fourier transform infra-red spectroscopy (FTIR) and room temperature ${ }^{57} \mathrm{Fe}$ Mossbauer spectroscopy. XRD was performed in a Bruker D8 Advance diffractometer with a graphite monochromator using $\mathrm{CuK}_{\alpha}$ radiation $(\lambda=1.5406 \AA)$. The patterns were collected within $10^{\circ}$ and $70^{\circ}$ in $2 \theta$ at a scan rate of $0.2(2 \theta) / \mathrm{min}$. The core crystalline size was calculated via the Scherrer equation (XRD size), using was obtained from the broadening (104) and (110) X-ray diffraction lines for the precursors, hematite and goethite, respectively and from (311) X-ray diffraction line for the resulting magnetite. FTIR was performed in a Bruker IFS 66V-S apparatus, in the range of $2000-250 \mathrm{~cm}^{-1}$. The samples were prepared for FTIR by diluting the dried powder in $\mathrm{KBr}$ at $2 \%$ by weight and pressing it into a pellet.

Room temperature ${ }^{57} \mathrm{Fe}$ Mössbauer spectroscopy was used to determine the resulting iron oxide phase after reduction of the precursors. Approximately $50 \mathrm{mg}$ of each sample was mixed with ca. $200 \mathrm{mg}$ sucrose in a pestle and mortar to form a paste, and then flattened within a coin shaped absorber. Spectra were recorded at room temperature in transmission geometry using a ${ }^{57} \mathrm{Co}$ source in a Rh matrix, using a W302 spectrometer and W202 detector, both from SEECo (Minneapolis, USA).

For the magnetic characterization, the samples were measured in powder form after drying in an inox coated oven at $50{ }^{\circ} \mathrm{C}$ for $24 \mathrm{~h}$. The powder was filled into polycarbonate capsules after accurately weighing. The sample mass was determined to be between 1 and $4 \mathrm{mg}$. Finally, the powder was immobilized in gelatin. The magnetization measurements have been performed in a Magnetic Property Measurement System (Quantum Design, USA) allowing the detection of the sample magnetic moment as a function of applied magnetic field and temperature.

In the field dependent measurements the magnetic field varied between-5 $\mathrm{T}$ and $5 \mathrm{~T}$. The amplitude of consecutive field steps was changed logarithmically to ensure a sufficient number of measurements at low fields. The time between consecutive magnetization measurements was about 3 min to guarantee quasi-static conditions. Hysteresis loops were measured at $295 \mathrm{~K}$ and at $5 \mathrm{~K}$. In the temperature dependent measurements the sample was first cooled down to
$5 \mathrm{~K}$ in zero magnetic field (zero field cooling, ZFC). Then, a magnetic field of $5 \mathrm{mT}$ was applied and the magnetic moment of the sample was measured with increasing temperature. After reaching $295 \mathrm{~K}$, the magnetic moment was measured with decreasing temperature under the presence of the magnetic field of $5 \mathrm{mT}$ (field cooling, FC).

Hysteresis loops were also measured in a vibrating sample magnetometer (MLVSM9, MagLab 9T, Oxford Instruments, UK) at room temperature and at $5 \mathrm{~K}$ after saturating the sample in a $5 \mathrm{~T}(-5 \mathrm{~T})$ field and recording the complete magnetization curves at a field change rate of $0.3 \mathrm{~T} / \mathrm{min}$.

\section{Results and discussion}

\subsection{Structural characterization of the precursors}

A general scheme of the synthesis strategy followed in this work is presented in Fig. 1. After the synthesis of the precursors and before the particles were coated, their morphology has been investigated by SEM. The scanning electron micrographs of the precursors (Fig. 2) show the three different morphologies obtained: (A) rhombohedra, (B) hexagonal discs and (C) needles. Image S1 (Supporting Information) confirms the morphology of the rhombohedra, where the typical square bipyramids of this polyhedron are observed in any of the orthogonal orientations of the nanoparticle. Both the size and the shape of the nanoparticles are highly homogeneous.

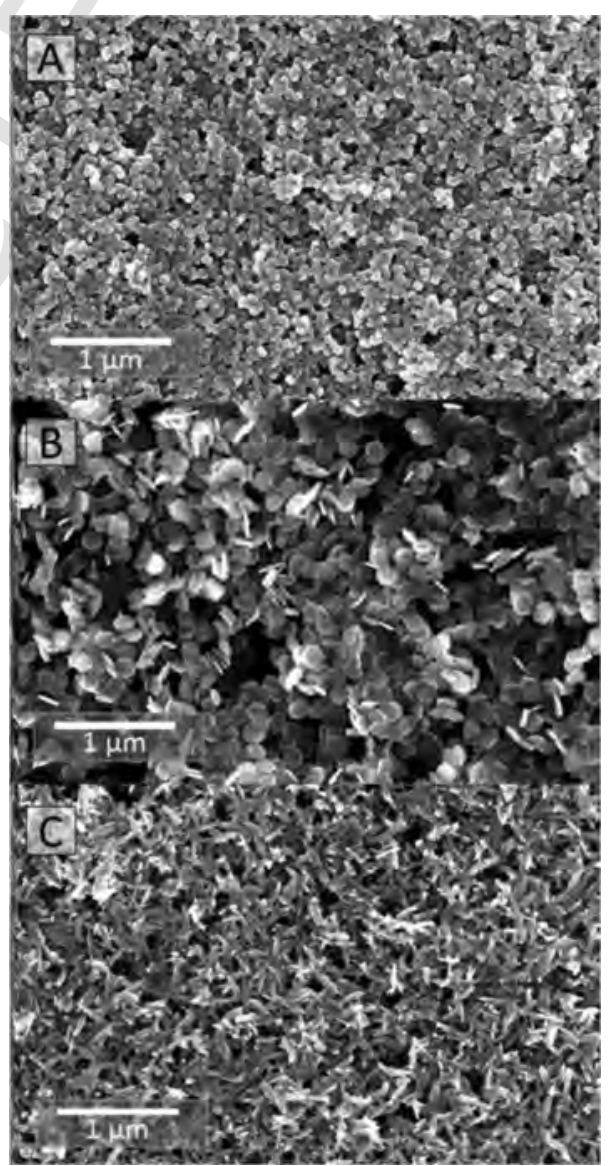

Fig. 2. SEM images of the precursor material (A) rhombohedra, (B) discs and (C) needles. 


\subsection{Transformation of the precursors to magnetite}

Fig. 3 shows TEM images of the precursor and the obtained MNPs where the core material has been transformed to magnetite by dry and wet reduction methods. The precursor has been successfully coated with a uniform silica shell around single cores or double-triple cores $(\sim 75 \%)$, although a fraction of multi-cores (5-10 cores coated) $(\sim 25 \%)$ cannot be discarded. TEOS condensation occurs only on the surface of the NPs and no colloidal silica nanoparticles have been observed.

The core size after reduction of the precursor was determined from the TEM images. The rhombohedra have an average core diameter of $67 \mathrm{~nm}$, the discs are $140 \mathrm{~nm}$ in diameter and $22 \mathrm{~nm}$ thick, and finally the needles are $183 \mathrm{~nm}$ in length and $33 \mathrm{~nm}$ thick. All samples have a standard deviation lower than $20 \%$ (see Table 1). By comparing the images before and after reduction we see that the particle size and morphology are conserved which is due to the silica coating of approximately $10 \mathrm{~nm}$. We also observe pores within the core structure after the reduction to magnetite, which is a consequence of the phase transformation from goethite in the case of the needles (Fig. 3 C), or hematite, in the case of the discs (Fig. 3B) and the rhombohedra (Fig. $3 \mathrm{~A})$.

When the silica coated goethite nanoparticles (either in powder or in solution) are heated up above $350{ }^{\circ} \mathrm{C}$, dehydration firstly occurs forming directly hematite:

$$
2 \mathrm{FeOOH} \rightarrow \mathrm{Fe}_{2} \mathrm{O}_{3}+\mathrm{H}_{2} \mathrm{O}
$$

The conversion of goethite to hematite is facilitated by the common anion structure shared by these two compounds. Three unit cells of goethite form one unit cell of hematite leading to contraction of the volume by a fraction of 0.62 [30]. The nanoparticles develop porosities within the structure due to the expulsion of water [22], occurring along the central axis of the needles, often merging with a single, elongated defect running along the entire length of the crystallite [31]. Pores along the c-axis were also reported [32] in good agreement with our observations. High resolution TEM images of the needles after the dehydration show contrast differences. These might be explained by either discontinuities of the thickness along the nanoparticle (surface roughness) or by discontinuities in the density of the nanoparticle (internal cavities). In order to elucidate the structure of the needles, a combination of under, over and in-focus high resolution TEM images have been taken. In doing this we are able to observe a certain degree of surface roughness, but more interestingly a porous internal structure has been evidenced (Fig. 4). Despite such porosity, a continuous single-crystalline structure corresponding to a monocrystal has been demonstrated for the needles. After the dehydration, the nanoparticles are reduced to magnetite without interparticle sintering and this is because of both the silica shell and the mild temperatures used for reduction (below $600{ }^{\circ} \mathrm{C}$ ).

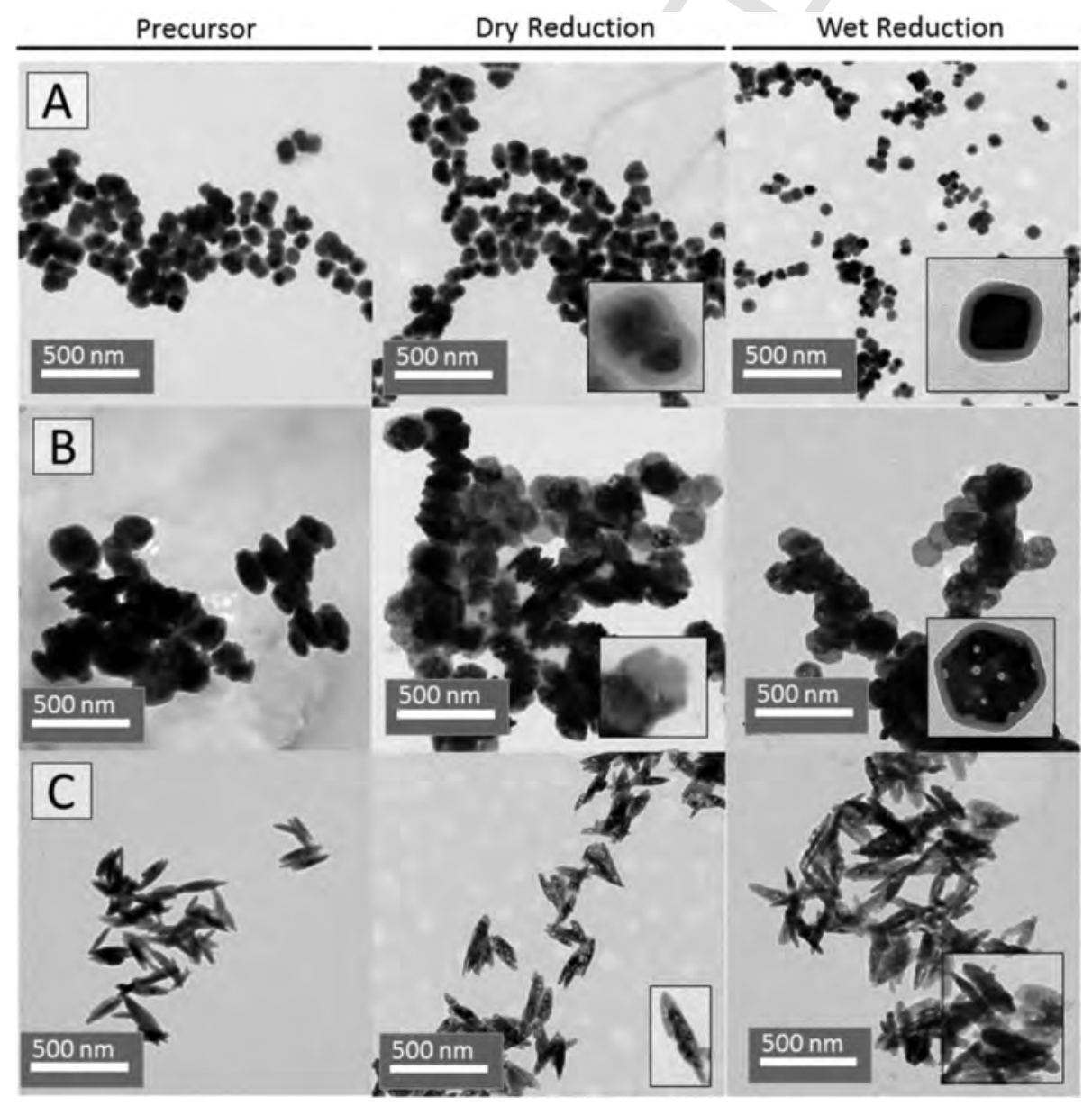

Fig. 3. TEM images of the precursors and the magnetic nanoparticles obtained by dry and wet reduction. (A) rhombohedra, (B) discs, (C) needles. 
Table 1

TEM core size vs. X-ray crystallite size of the hematite/goethite precursor and the obtained MNPs by wet and dry reduction. P: Antiferromagnetic Precursor, W.R.: Wet Reduction, D.R.: Dry Reduction.

\begin{tabular}{|c|c|c|c|c|c|c|}
\hline \multirow[t]{2}{*}{ Sample } & \multicolumn{3}{|c|}{ Antiferromagnetic precursors } & \multicolumn{3}{|c|}{ After reduction } \\
\hline & Iron Oxide & $\begin{array}{l}\overline{\boldsymbol{D}} \text { TEM } \\
(\mathrm{nm}), \\
\sigma(\%)\end{array}$ & $\underset{(\mathrm{Dm})}{\underset{X R D}{P}}$ & $\begin{array}{l}\text { Iron } \\
\text { Oxide }\end{array}$ & $\begin{array}{l}\bar{D} \underset{X R D}{W . R} \\
(\mathrm{~nm})\end{array}$ & $\begin{array}{l}\bar{D} \underset{X R D}{D \cdot R} \\
(\mathrm{~nm})\end{array}$ \\
\hline Rhombohedra & $\alpha-\mathrm{Fe}_{2} \mathrm{O}_{3}$ & $67, \sigma=18$ & 69 & $\mathrm{Fe}_{3} \mathrm{O}_{4}$ & 47 & 48 \\
\hline Discs & $\alpha-\mathrm{Fe}_{2} \mathrm{O}_{3}$ & $\begin{array}{r}140 \times 22 \\
\sigma=10,4\end{array}$ & 121 & $\mathrm{Fe}_{3} \mathrm{O}_{4}$ & 34 & 42 \\
\hline Needles & $\alpha-\mathrm{FeO}(\mathrm{OH})$ & $\begin{array}{r}183 \times 33 \\
\sigma=18,7\end{array}$ & 17 & $\mathrm{Fe}_{3} \mathrm{O}_{4}$ & 19 & 27 \\
\hline
\end{tabular}

TEM images obtained in this manner reveal the presence of pores for all particle morphologies, synthesised from both types of precursor. For hematite precursors, we observe pores in both the magnetite discs and rhombohedra that are randomly distributed within the structure (Fig. 3), as previously observed by Edwards et al. [33]. The reduction itself is associated with the formation of tunnels by the removal of oxygen. Magnetite is formed in the surrounding areas parallel to the tunnel generation, which yields an overall crystal structure of magnetite with such pores [34].

High-resolution TEM (HR-TEM) images of the needles and rhombohedra after both the wet and dry reduction are shown in Figs. S2-S4 (supporting information). For the rhombohedra, Fourier transformation of the HR-TEM image yields a diffraction pattern that corresponds to a monocrystal and demonstrates that even with such pores, each particle has a single crystal structure. It is particularly striking in the case of needles (Fig. S3, Supporting Information) after the dehydration and reduction process, that a continuous single-crystal structure corresponding to a monocrystal has been likewise demonstrated, despite the porosity along the c-axis. However, when lower reduction temperatures and shorter times were used, nanorods consisting of clusters of maghemite embedded in an aniferromagnetic hematite matrix were obtained [35].

\subsection{Identification of iron oxide phase and measurement of core size}

XRD patterns confirm that the precursor used to synthesize the rhombohedra and discs is indeed hematite, whilst the diffraction pattern of the precursor used to synthesize needles corresponds to goethite (Fig. 5). The X-ray diffractograms of nanoparticles produced via wet and dry reduction methods indicate a single iron oxide phase, which we attribute to the inverse spinel structure of either magnetite or maghemite. We have used the Scherrer equation on the most intense diffraction peak to calculate the average core crystalline size of each of the precursors; this corresponds to the (110) reflection for hematite and the (111) reflection for goethite. XRD patterns confirm that the cores of both the hematite rhombohedra and discs are single crystal structures and have similar average sizes, in good agreement with that measured via TEM. For the goethite needles, the core crystalline size obtained using the (111) reflection is closer to the smallest TEM dimension, and is in agreement with the goethite crystals being elongated along the (100) direction [22]. Table 1 shows core sizes determined by TEM and crystal sizes obtained by XRD measurements.

FTIR spectra of the hematite precursors (Fig. S5 in supporting information) show bands at $355 \mathrm{~cm}^{-1}$ and $477 \mathrm{~cm}^{-1}$ which are attributed to the parallel and transverse vibrations $\left(\mathrm{E}_{\mathrm{u}}\right)$, and bands at $575 \mathrm{~cm}^{-1}$ and $381 \mathrm{~cm}^{-1}$ attributed to the longitudinal vibration $\left(\mathrm{A}_{2 \mathrm{u}}\right)$. For the goethite precursor, a band at $409 \mathrm{~cm}^{-1}$ can be attributed to the $\mathrm{Fe}-\mathrm{O}$ antisymmetric stretch parallel to the c-axis and bands at 628,790 , $886 \mathrm{~cm}^{-1}$ can be attributed to the $\mathrm{Fe}-\mathrm{O}$ symmetric stretch parallel to the a-axis.

The transformation of the antiferromagnetic precursors to the magnetic phase was monitored using a combination of XRD - via the (311) reflection for the obtained magnetite nanoparticles - and FTIR. It should be mentioned that there are two main features contributing to the X-ray broadening, the crystal order and the particle shape, which makes such an analysis via XRD difficult.

The crystallite sizes obtained from the (311) reflection for the resulting magnetite needles, which is at $30^{\circ}$ of the longest particle dimension, indicates a slightly increase with respect to the crystalline size calculated for the precursor, indicating a somewhat better crystal order after the reduction. By comparing XRD values for both reduction techniques, it seems that the dry reduction provides particles with a slightly larger crystallite size or less defects than the wet reduction.

Infra-red (IR) spectra measured after both wet and dry reduction, show bands that correspond to the silica coating of the nanoparticles and bands corresponding to magnetite, together with the band at $1632 \mathrm{~cm}^{-1}$ due to the stretching and bending vibration of $\mathrm{H}_{2} \mathrm{O}$ molecules. The very strong and broad IR band at $1111 \mathrm{~cm}^{-1}$ with a shoulder at $1188 \mathrm{~cm}^{-1}$ is usually assigned to the transversal optical (TO) and longitudinal optical (LO) modes of the Si-O-Si asymmetric stretching vibrations, the band at $956 \mathrm{~cm}^{-1}$ is assigned to silanol groups and the one at $800 \mathrm{~cm}^{-1}$ is assigned to $\mathrm{Si}-\mathrm{O}-\mathrm{Si}$ symmetric stretching vibrations. Lastly, in the Fe-O range IR bands at $568 \mathrm{~cm}^{-1}$ and $357 \mathrm{~cm}^{-1}$ are attributable to magnetite [22], (Fig. S5). The pres

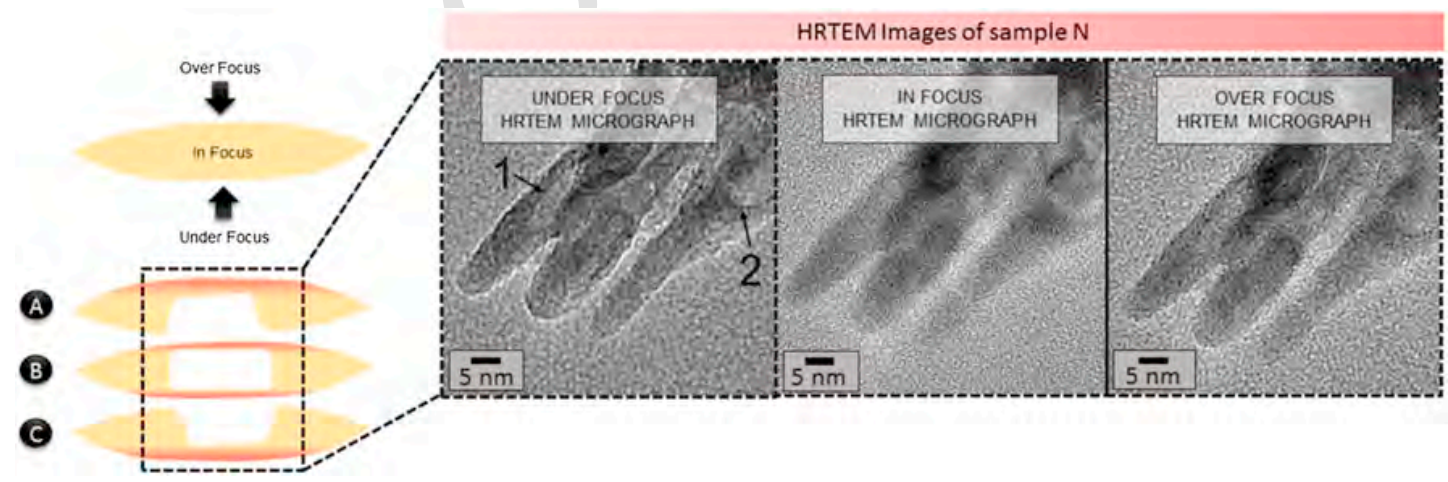

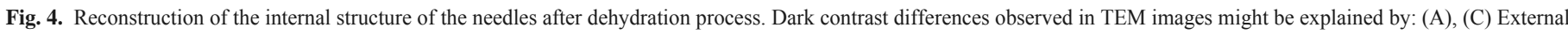

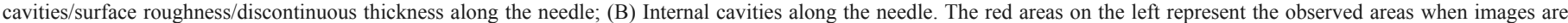
taken under, over and in-focus. (For interpretation of the references to colour in this figure legend, the reader is referred to the web version of this article.) 


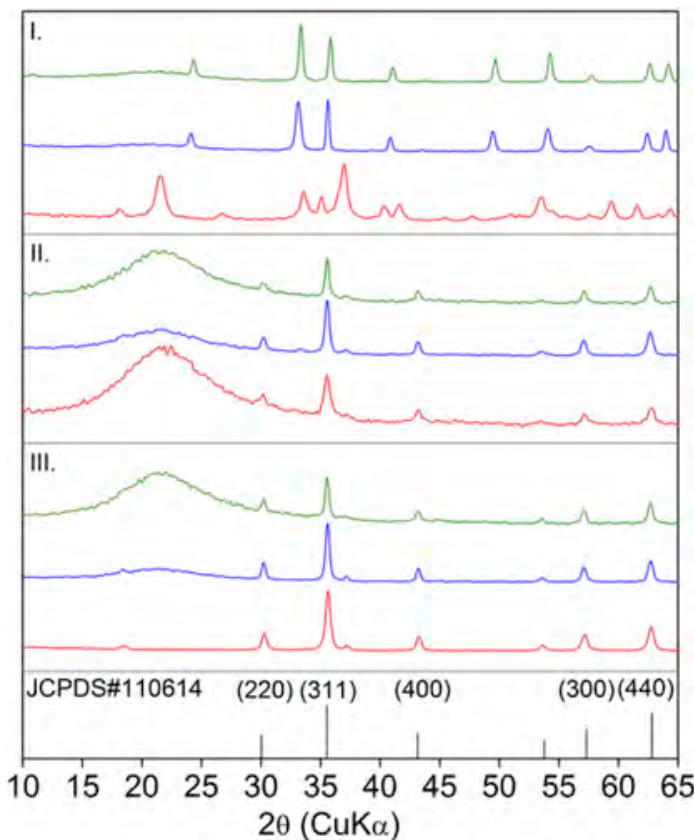

Fig. 5. X-ray powder diffractograms of the precursor (I) and products obtained by wet (II) and dry reduction (III). A single phase, whose Bragg peaks are attributed to magnetite or maghemite, is obtained through both reduction techniques for all morphologies: rhombohedra (green), discs (blue) and needles (red). (For interpretation of the references to colour in this figure legend, the reader is referred to the web version of this article.)

ence of maghemite is not evident due to the absence of small shoulders around these two main bands in the low frequency range which are particularly sharp for the needles.

Room temperature Mössbauer spectra of rhombohedra and needles reduced via dry powder methods are shown in Fig. 6. For the rhombohedra, the spectrum is a sharp six-line pattern comprising two sub-spectra and is reminiscent of stoichiometric magnetite. The best fit spectra comprise a superposition of three Voigtian sextets, two of which have isomer shifts close to $0.26 \mathrm{~mm} / \mathrm{s}$ and $0.67 \mathrm{~mm} / \mathrm{s}$, and associated hyperfine fields close to $49 \mathrm{~T}$ and $46 \mathrm{~T}$, respectively, which are indicative of the tetra and octahedral sites within magnetite.

The third sub-component comprises less than $8 \%$ of the spectral area and is a broad sextet, with an isomer shift of $0.26 \mathrm{~mm} / \mathrm{s}$ and is likely to come from $\mathrm{Fe}$ atoms around pore structures in the rhombo hedra. We surmise that majority of the sample is well crystallised, stoichiometric magnetite.

Whilst we see that the Mössbauer spectrum of the needles (Fig. 6 (b)) also comprises a 6-line pattern, here we see that the absorption lines are broader and also slightly asymmetric. The best fit is also obtained using a superposition of three Voigtian sextets, two of which have isomer shifts and hyperfine fields reminiscent of magnetite as seen for the rhombohedra. The third sub-component comprises ca. $18 \%$ of the spectral area and results in the slight 'hanging' of the spectra at $\mathrm{v}=0$. The isomer shift of this component is $0.41 \mathrm{~mm} / \mathrm{s}$ and cannot be attributed to either $\mathrm{Fe}^{3+}$ on the A site of $\mathrm{Fe}^{2+, 3+}$ on the $\mathrm{B}$ site. We attribute this component to the highly porous structure disrupting the crystalline structure experienced by Fe nuclei in the needles. The mean isomer shift of the spectra is $0.48 \mathrm{~mm} / \mathrm{s}$ and $0.49 \mathrm{~mm} /$ $\mathrm{s}$, respectively, which are close to the value of $0.53 \mathrm{~mm} / \mathrm{s}$ for stoichiometric magnetite [36,37].

\subsection{Magnetic characterization of the magnetite particles}

The field and temperature dependent magnetization measurements on all samples are shown in Fig. 7. For each sample system, the field dependent measurements performed at $5 \mathrm{~K}$ and $295 \mathrm{~K}$ are plotted in one graph. The insets show the magnetization behaviour in the low field regime at $\pm 300 \mathrm{kA} / \mathrm{m}$.

All magnetization curves show similar behaviour for different shapes and different reduction techniques. The samples exhibit non-zero remanence and coercivity indicating that the particles are magnetically blocked at room temperatures and below.

Looking into the details, it can be seen that the rhombohedra and needles obtained by wet reduction present a so-called wasp-waisted hysteresis loop, which is not observed in the materials produced by dry reduction. Roberts et al. explained the occurrence of a wasp-waisted hysteresis loop by a mixture of single- and multi-domain remanence states within the particles [38]. Since the samples prepared by dry reduction do not show the wasp-waisted hysteresis behaviour, it seems that this reduction technique produces particles with a more uniform magnetization state compared to the particles synthesised by wet reduction. This is in good agreement with the findings on the crystallite size measured by XRD, because defects and pores are usually responsible for the pinning and nucleation sites for magnetic domains, and obviously these defects and pores are less pronounced for dry reduced MNPs.

The saturation magnetization of the different samples is determined at $5 \mathrm{~T}$ where nearly all magnetic moments are aligned in field

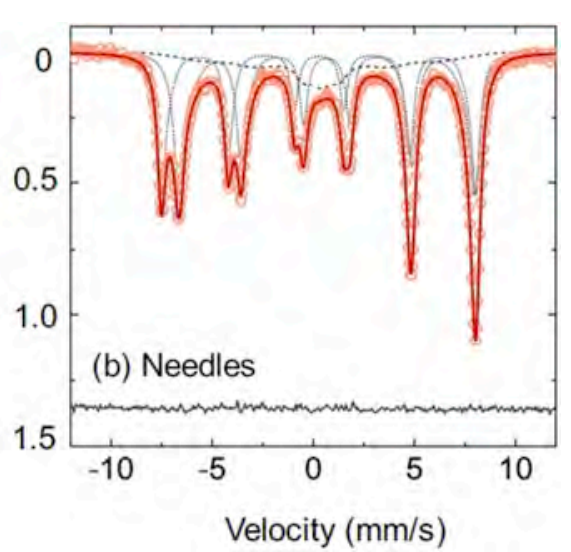

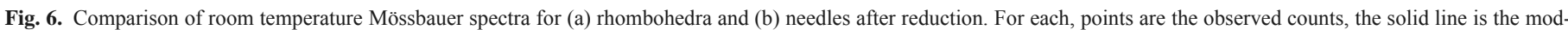
elled best fit to the data, and the dotted lines correspond to the Voigtian sub spectra. The quality of fit is indicated by the reduced $\chi^{2}$ and the residual, given by $\mathrm{I}_{\text {obs }}-\mathrm{I}_{\text {cal }}$. 

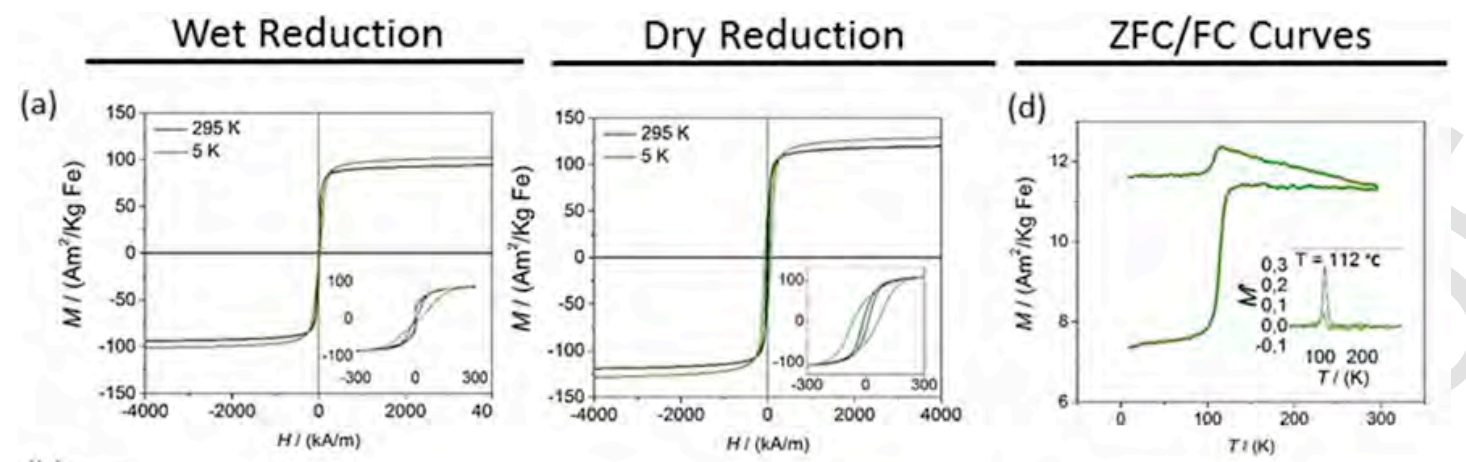

(b)
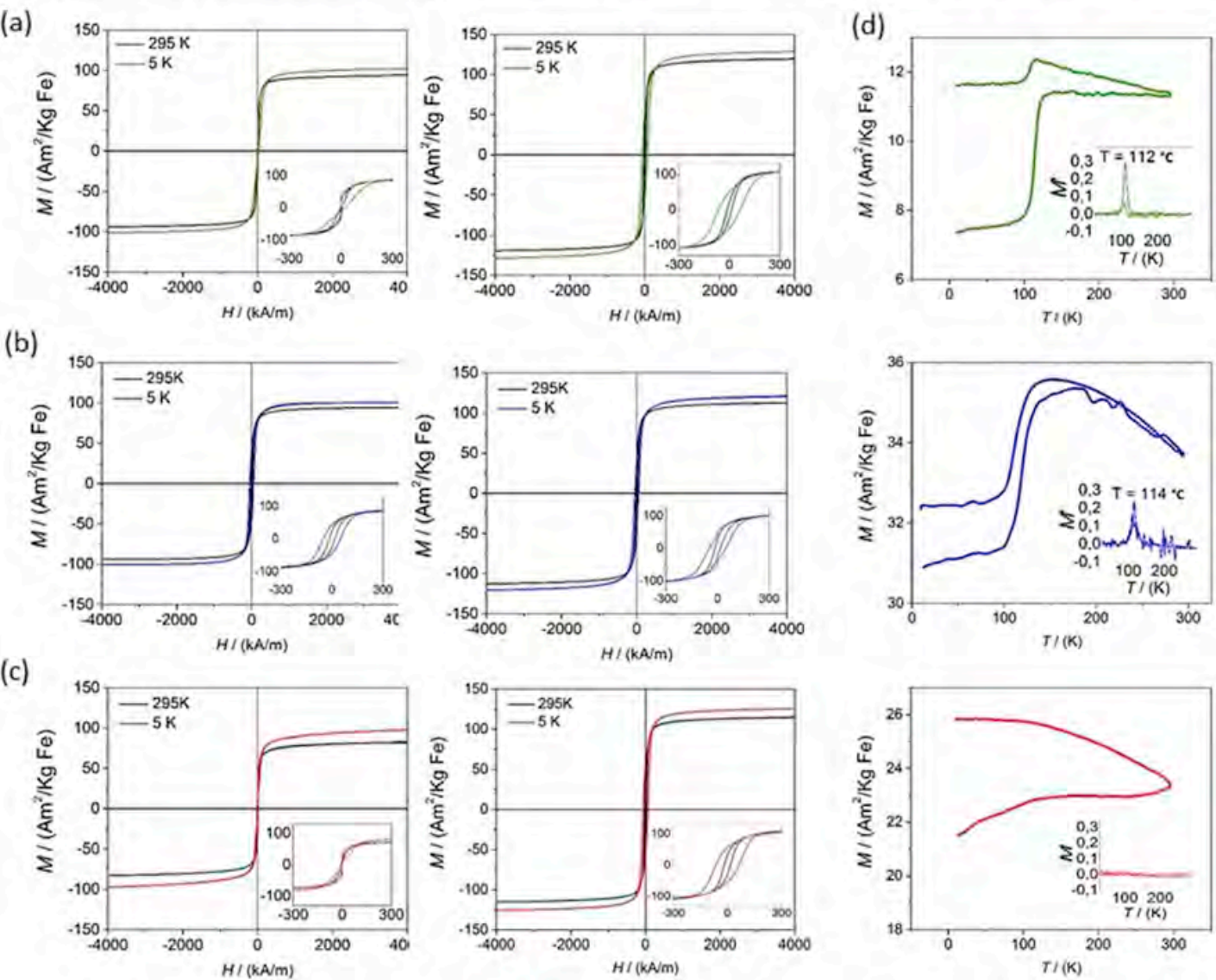

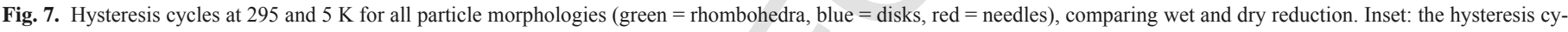

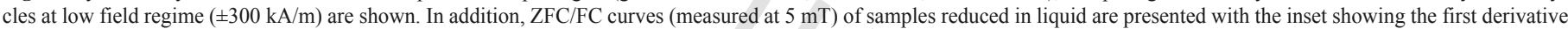
of the magnetization with respect to temperature. (For interpretation of the references to colour in this figure legend, the reader is referred to the web version of this article.)

direction and thus dipolar interactions between the particle's magnetic moments are negligible. The saturation magnetization increases with decreasing temperature and the values of the saturation magnetization at room temperature are smaller than the $130 \mathrm{Am}^{2} / \mathrm{kg}(\mathrm{Fe})$ found for bulk magnetite [39]. The reduced magnetization for fine-particle systems by finite-size effects is well known in literature [40]. Magnetite nanocrystals obtained directly by the polyol process and aggregated in dense or hollow spheres show similar saturation magnetization values $\left(110 \mathrm{Am}^{2} / \mathrm{kg}(\mathrm{Fe})\right)$ and a coercive field of $(11 \mathrm{kA} / \mathrm{m})$ at room temperature [11].

The saturation magnetization $M_{\mathrm{S}}$ and coercive field $H_{\mathrm{C}}$ have been extracted from the magnetization data and summarized in Table 2 . The value of $M_{\mathrm{S}}$ is largest for the rhombohedra while the discs exhibit the lowest saturation magnetization and that can be traced back to influences of the particles size. Interestingly, the saturation magnetization is considerably lower for the wet reduced particles than for dry reduction indicating again a significant effect of the reduction process and the presence of pores on the magnetic properties of the material. For the disc shaped particles, the coercive field is identical in the wet and in the dry reduced samples. The coercivity of the rhombohedra and the needles cannot be compared due to the wasp-waisted hysteresis behaviour in case of wet reduction.

Field dependent magnetization curves recorded at higher field sweeping rates for the rhombohedra and needles are shown in Fig. 8.
Table 2

Comparison of the values of saturation magnetization (MS) of magnetite particles after wet and dry reduction, and coercivity (HC) at 5 and $295 \mathrm{~K}$. (R) indicates the rhombohedra, (D) the discs and (N) the needles particles.

\begin{tabular}{lllllllll}
\hline Sample & \multicolumn{1}{l}{ Wet reduction } & \multicolumn{7}{l}{ Dry reduction } \\
\hline & $\mathrm{M}_{\mathrm{S}}\left(\mathrm{Am}^{2} / \mathrm{kg}(\mathrm{Fe})\right)$ & $\mathrm{H}_{\mathrm{C}}(\mathrm{kA} / \mathrm{m})$ & \multicolumn{2}{l}{$\mathrm{M}_{\mathrm{S}}\left(\mathrm{Am}^{2} / \mathrm{kg}(\mathrm{Fe})\right)$} & $\mathrm{H}_{\mathrm{C}}(\mathrm{kA} / \mathrm{m})$ \\
\hline & $5 \mathrm{~K}$ & $295 \mathrm{~K}$ & $5 \mathrm{~K}$ & $295 \mathrm{~K}$ & $5 \mathrm{~K}$ & $295 \mathrm{~K}$ & $5 \mathrm{~K}$ & $295 \mathrm{~K}$ \\
\hline Rhombohedra & 102 & 94 & 11 & 4 & 128 & 119 & 62 & 12 \\
Discs & 100 & 94 & 50 & 17 & 121 & 113 & 50 & 15 \\
Needles & 98 & 83 & 0 & 4 & 126 & 115 & 62 & 16 \\
\hline
\end{tabular}

Here the wasp-waisted hysteresis curve as observed in quasi-static magnetization measurements has vanished. The fact that the hysteresis curve depends on the field change rate indicates that the wasp-waisted hysteresis curves originate from domain formation within the particles, since the nucleation of domain wall and its movement are time dependent [41].

Zero-field cooled/field cooled (ZFC/FC) magnetization curves of all products obtained from wet reduction are shown in (Fig. 7). A clear Verwey transition [42] is observed in the case of rhombohedra and discs at 112-114 K (obtained from a hematite precursor). However, the transition in case of the discs is less sharp than for the rhom 
(a)

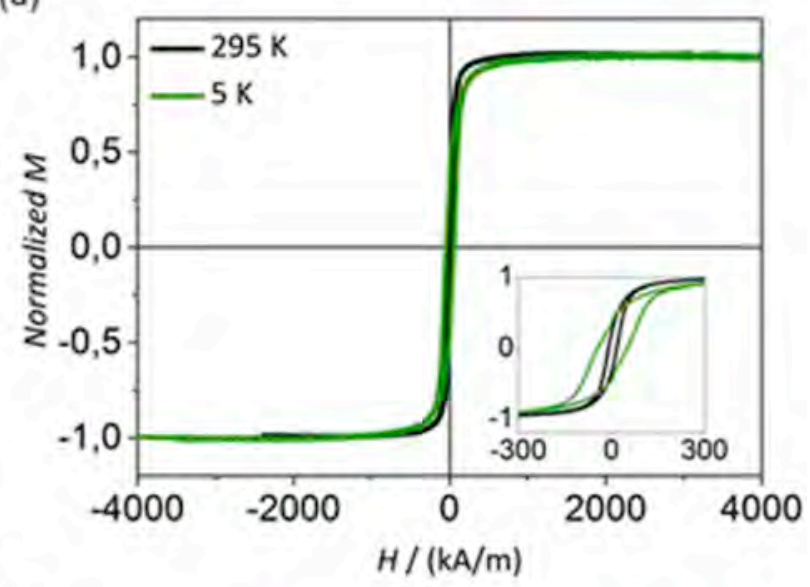

(b)

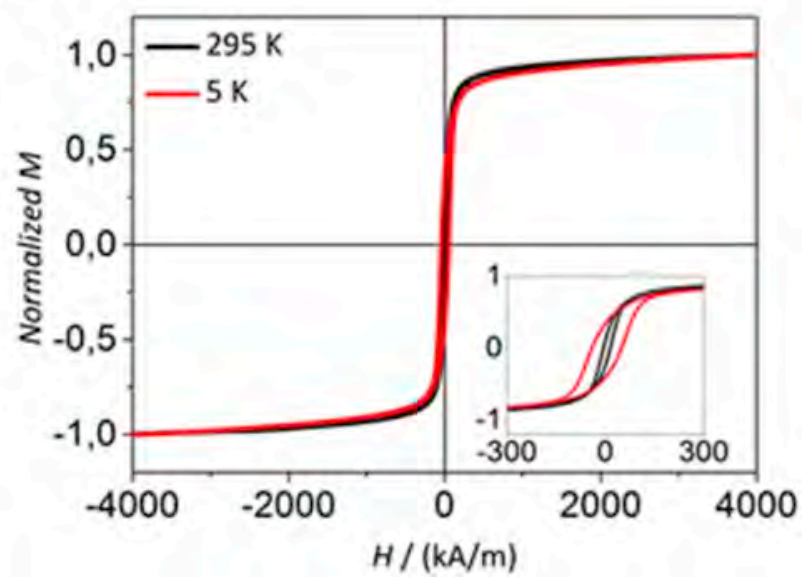

Fig. 8. Magnetization curves between $\pm 5 \mathrm{~T}$ measured with a field sweep of $0.3 \mathrm{~T} / \mathrm{min}$ for magnetite rhombohedra (green) and needles (red) obtained by wet reduction. (For interpretation of the references to colour in this figure legend, the reader is referred to the web version of this article.)

bohedra. In case of the needles (obtained from a goethite precursor) the Verwey transition is almost invisible.

Small degrees of non-stoichiometry or impurities in magnetite can reduce the Verwey transition temperature [43], located at $125 \mathrm{~K}$ for stoichiometric magnetite [44] and that could explain the different Verwey temperature observed for rhombohedra and discs. However, our XRD and Mössbauer studies confirm the magnetite nature of the particles. In fact, the reason for the shifted Verwey transition could be the crystal size, as discussed in Refs. [42]' [45], where the state change during the transition and its temperature reduces with decreasing crystal size. The fact that almost no transition is observed in the needles could be associated to the smaller crystal size as determined by XRD for this sample (Table 1) and the observation of a porous structure within a single particle by HRTEM (Fig. 5 and Fig. S2 in supporting information). Móssbauer spectra support this conclusion showing broader lines and a central singlet for the needles suggesting poorer crystallinity, which would explain the loss of the Verwey transition, against sharp absorption lines for rhombohedra corresponding to pure stoichiometric magnetite.

The shape of the Verwey transition for the different morphologies, especially for the smeared out transition of the discs, could be traced back to the existence of pores inside the particles and therefore a less-ordered crystal structure.

\section{Conclusions}

In this work, an alternative route to produce a library of iron oxide magnetic nanoparticles in a core size range of 50-100 $\mathrm{nm}$ and three different shapes has been successfully demonstrated. Here the goethite and hematite precursors are coated with silica to avoid sintering and to conserve the particle shape in the subsequent reduction of the precursor material to magnetite. Two different reduction methods have been successfully applied yielding a single iron oxide phase of magnetite.

The magnetite phase has been verified by X-ray diffractograms and ZFC/FC magnetization curves. Magnetite nanoparticles coming from hematite precursor show a very clear Verwey transition at slightly lower temperatures than the reported ones for pure magnetite, probably due to small impurities or pores in the particles. The hysteresis parameters revealed that there is a difference between the magnetic material obtained by wet and dry reduction with higher values of saturation magnetization after dry reduction. However, rhombohedra and needle shaped samples show, in the case of the wet reduction, interesting magnetic properties with a wasp-shaped hysteresis curve and a very low coercive field at room temperature originated probably from the domain formation within the particles.

In addition, the approach for the reduction of the precursors has an influence on the magnetic behaviour of the final magnetite particles. MNPs that have been produced by dry reduction show fewer defects in the crystal structure. The possibility of generating a discontinuous structure within a particle by forcing the pore formation may be an interesting strategy to develop new materials with tuned magnetic properties for biomedical applications.

In conclusion, we demonstrate the production of large $(>25 \mathrm{~nm})$ magnetite MNPs where shape and internal structure could be varied by the synthesis parameters. We demonstrate that both MNPs shape and internal MNPs structure affect strongly the magnetic behaviour of the MNPs. In future characterization of magnetite MNPs systems these parameters should always be taken into account.

\section{Supporting information description}

Additional data on High-Resolution Transmission Electron Microscopy and Infrared Spectroscopy are available.

\section{Acknowledgements}

We would like to thank Dr. Teresita González-Carreño for her lab experiment assistance. This work was supported by the EC FP-7 grant "NanoMag" (grant agreement no 604448) and the Spanish Government by MAGO project (MAT2014-52069-R). The magnetic characterization was supported by COST Action TD1402. LG acknowledges financial support from the Ramón y Cajal subprogram (RYC-2014-15512).

\section{Appendix A. Supplementary data}

Supplementary data related to this article can be found at http://dx. doi.org/10.1016/j.actamat.2016.12.016.

\section{References}

[1] R. Mejías, S. Pérez-Yagüe, L. Gutiérrez, L.I. Cabrera, R. Spada, P. Acedo, C.J. Serna, F.J. Lázaro, T. Villanueva, M.D.P. Morales, D.F. Barber, Dimercaptosuccinic acid-coated magnetite nanoparticles for magnetically guided in vivo delivery of interferon gamma for cancer immunotherapy, Biomaterials 32 (11) (2011) 2938-2952. 
[2] K. Maier-Hauff, F. Ulrich, D. Nestler, H. Niehoff, P. Wust, B. Thiesen, H. Orawa, V. Budach, A. Jordan, Efficacy and safety of intratumoral thermotherapy using magnetic iron-oxide nanoparticles combined with external beam radiotherapy on patients with recurrent glioblastoma multiforme, J. Neuro-Oncology 103 (2) (2010) 317-324.

[3] T.-H. Shin, Y. Choi, S. Kim, J. Cheon, Recent advances in magnetic nanoparticle-based multi-modal imaging, Chem. Soc. Rev. 44 (14) (2015) 4501-4516.

[4] A.P. Khandhar, R.M. Ferguson, H. Arami, K.M. Krishnan, Monodisperse magnetite nanoparticle tracers for in vivo magnetic particle imaging, Biomaterials 34 (15) (2013) 3837-3845.

[5] I. Hilger, W.A. Kaiser, Iron oxide-based nanostructures for MRI and magnetic hyperthermia, Nanomedicine 7 (9) (2012) 1443-1459.

[6] Y.X.J. Wang, J.-M. Idee, C. Corot, Scientific and industrial challenges of developing nanoparticle-based theranostics and multiple-modality contrast agents for clinical application, Nanoscale 7 (39) (2015) 16146-16150.

[7] D. Silvio, H. Rudolf, Magnetic particle hyperthermia - a promising tumour therapy?, Nanotechnology 25 (45) (2014) 452001

[8] L.n. Lartigue, P. Hugounenq, D. Alloyeau, S.P. Clarke, M. Lévy, J.-C. Bacri, R. Bazzi, D.F. Brougham, C. Wilhelm, F. Gazeau, Cooperative organization in iron oxide multi-core nanoparticles potentiates their efficiency as heating mediators and MRI contrast agents, ACS Nano 6 (12) (2012) 10935-10949.

[9] S. Dutz, Are magnetic multicore nanoparticles promising candidates for biomedical applications?, IEEE Trans. Magn. 52 (9) (2016) 1-3.

[10] D. Serantes, K. Simeonidis, M. Angelakeris, O. Chubykalo-Fesenko, M. Marciello, M.d.P. Morales, D. Baldomir, C. Martinez-Boubeta, Multiplying magnetic hyperthermia response by nanoparticle assembling, J. Phys. Chem. C 118 (11) (2014) 5927-5934.

[11] N. Guan, Y. Wang, D. Sun, J. Xu, A simple one-pot synthesis of single-crystalline magnetite hollow spheres from a single iron precursor, Nanotechnology 20 (10) (2009) 105603.

[12] B. Jia, L. Gao, Morphological transformation of Fe3O4 spherical aggregates from solid to hollow and their self-assembly under an external magnetic field, J. Phys. Chem. C 112 (3) (2008) 666-671.

[13] G. Salas, C. Casado, F.J. Teran, R. Miranda, C.J. Serna, M.P. Morales, Controlled synthesis of uniform magnetite nanocrystals with high-quality properties for biomedical applications, J. Mater. Chem. 22 (39) (2012) 21065-21075.

[14] D. Kim, N. Lee, M. Park, B.H. Kim, K. An, T. Hyeon, Synthesis of uniform ferrimagnetic magnetite nanocubes, JACS 131 (2) (2009) 454- 455.

[15] M.V. Kovalenko, M.I. Bodnarchuk, R.T. Lechner, G. Hesser, F. Schäffler, W Heiss, Fatty acid salts as stabilizers in size- and shape-controlled nanocrystal Synthesis: the case of inverse spinel iron oxide, JACS 129 (20) (2007) 6352-6353.

[16] S. Palchoudhury, W. An, Y. Xu, Y. Qin, Z. Zhang, N. Chopra, R.A. Holler, C.H. Turner, Y. Bao, Synthesis and growth mechanism of iron oxide nanowhiskers, Nano Lett. 11 (3) (2011) 1141-1146.

[17] H. Sun, B. Chen, X. Jiao, Z. Jiang, Z. Qin, D. Chen, Solvothermal synthesis of tunable Electroactive magnetite nanorods by controlling the side reaction, J. Phys. Chem. C 116 (9) (2012) 5476-5481.

[18] L. Zhang, J. Wu, H. Liao, Y. Hou, S. Gao, Octahedral Fe3O4nanoparticles and their assembled structures, Chem. Commun. (29) (2009) 4378-4380.

[19] J. Lu, X. Jiao, D. Chen, W. Li, Solvothermal synthesis and characterization of Fe3O4 and $\gamma$-Fe2O3 nanoplates, J. Phys. Chem. C 113 (10) (2009) 4012-4017.

[20] Y. Zeng, R. Hao, B. Xing, Y. Hou, Z. Xu, One-pot synthesis of Fe3O4 nanoprisms with controlled electrochemical properties, Chem. Commun. 46 (22) (2010) 3920-3922.

[21] A.F. Rebolledo, O. Bomatí-Miguel, J.F. Marco, P. Tartaj, A facile synthetic route for the preparation of superparamagnetic iron oxide nanorods and nanorices with tunable surface functionality, Adv. Mater 20 (9) (2008) $1760-1765$.

[22] R.M. Cornell, U. Schwertmann, The Iron Oxides: Structure, Properties, Reactions, Occurrences and Uses, John Wiley \& Sons, 2003.
[23] C. Graf, D.L.J. Vossen, A. Imhof, A. van Blaaderen, A general method to coat colloidal particles with silica, Langmuir 19 (17) (2003) 6693-6700.

[24] W. Stöber, A. Fink, E. Bohn, Controlled growth of monodisperse silica spheres in the micron size range, J. Colloid Interface Sci. 26 (1) (1968) 62-69.

[25] M.P. Morales, C. Pecharroman, T.G. Carreñ, C.J. Serna, Structural characteristics of uniform $\gamma$-Fe2O3 particles with different axial (Length/Width) ratios, J. Solid State Chem. 108 (1) (1994) 158-163.

[26] Y. Yang, X. Liu, Y. Lv, T.S. Herng, X. Xu, W. Xia, T. Zhang, J. Fang, W. Xiao, J. Ding, Orientation mediated enhancement on magnetic hyperthermia of Fe3O4 nanodisc, Adv. Funct. Mater 25 (5) (2015) 812-820.

[27] Y. Yang, X. Liu, J. Ding, Synthesis of a-Fe2O3 templates via Hydrothermal route and $\mathrm{Fe} 3 \mathrm{O} 4$ particles through subsequent chemical reduction, Sci. Adv. Mater. 5 (9) (2013) 1199-1207.

[28] R. Pozas, M. Ocaña, M.P. Morales, C.J. Serna, Uniform nanosized goethite particles obtained by aerial oxidation in the FeSO4-Na2CO3 system, J. Colloid Interface Sci. 254 (1) (2002) 87-94.

[29] L.M. Liz-Marzán, P. Mulvaney, The assembly of coated nanocrystals, J. Phys. Chem. B 107 (30) (2003) 7312-7326.

[30] H. Naono, K. Nakai, T. Sueyoshi, H. Yagi, Porous texture in hematite derived from goethite: mechanism of thermal decomposition of geothite, J. Colloid Interface Sci. 120 (2) (1987) 439-450.

[31] A.S.J. Baker, A.S.C. Brown, M.A. Edwards, J.S.J. Hargreaves, C.J. Kiely, A. Meagher, Q.A. Pankhurst, A structural study of haematite samples prepared from sulfated goethite precursors: the generation of axial mesoporous voids, J. Mater. Chem. 10 (3) (2000) 761-766

[32] J. Morales, J.L. Tirado, M. Macias, Changes in crystallite size and microstrains of hematite derived from the thermal decomposition of synthetic akaganeite, J. Solid State Chem. 53 (3) (1984) 303-312.

[33] H. Brill-Edwards, B.L. Daniell, R.L. Samuel, Structural changes accompanying the reduction of polycrystalline haematite, J. Iron Steel Inst. 203 (1965). 361-\&.

[34] P. Swann, N. Tighe, High voltage microscopy of the reduction of hematite to magnetite, Metall. Trans. B 8 (2) (1977) 479-487.

[35] A.F. Rebolledo, S. Laurent, M. Calero, A. Villanueva, M. Knobel, J.F. Marco, P. Tartaj, Iron oxide nanosized clusters embedded in porous nanorods: a new colloidal Design to Enhance capabilities of MRI contrast agents, ACS Nano 4 (4) (2010) 2095-2103.

[36] G.M. da Costa, C. Blanco-Andujar, E. De Grave, Q.A. Pankhurst, Magnetic nanoparticles for in vivo use: a critical assessment of their composition, J. Phys. Chem. B. 118 (40) (2014) 11738-11746.

[37] J. Santoyo Salazar, L. Perez, O. de Abril, L. Truong Phuoc, D. Ihiawakrim, M. Vazquez, J.-M. Greneche, S. Begin-Colin, G. Pourroy, Magnetic iron oxide nanoparticles in 10-40 $\mathrm{nm}$ range: composition in terms of magnetite/maghemite ratio and effect on the magnetic properties, Chem. Mater 23 (6) (2011) 1379-1386

[38] A.P. Roberts, Y. Cui, K.L. Verosub, Wasp-waisted hysteresis loops: mineral magnetic characteristics and discrimination of components in mixed magnetic systems, J. Geophys. Res. Solid Earth 100 (B9) (1995) 17909-17924.

[39] R.C. O'handley, Modern Magnetic Materials: Principles and Applications, Wiley New York, 2000.

[40] X. Batlle, A. Labarta, Finite-size effects in fine particles: magnetic and transport properties, J. Phys. D. Appl. Phys. 35 (6) (2002). R15.

[41] B. Hillebrands, K. Ounadjela, Spin Dynamics in Confined Magnetic Structures I, Springer, 2001.

[42] G. Goya, T. Berquo, F. Fonseca, M. Morales, Static and dynamic magnetic properties of spherical magnetite nanoparticles, J. Appl. Phys. 94 (5) (2003) 3520-3528.

[43] R. Aragón, D.J. Buttrey, J.P. Shepherd, J.M. Honig, Influence of nonstoichiometry on the Verwey transition, Phys. Rev. B 31 (1) (1985) 430-436.

[44] W. Friedrich, The Verwey transition - a topical review, J. Phys. Condens. Matter 14 (12) (2002). R285.

[45] C.L. Snow, Q. Shi, J. Boerio-Goates, B.F. Woodfield, Heat capacity studies of nanocrystalline magnetite (Fe3O4), J. Phys. Chem. C 114 (49) (2010) 21100-21108. 